\title{
Isolation and characterization of in vitro culture of hair follicle cells differentiated from umbilical cord blood mesenchymal stem cells
}

\author{
ZHANG-YU BU, LI-MIN WU, XIAO-HONG YU, JIAN-BO ZHONG, PING YANG and JIAN CHEN \\ Department of Dermatology, Hangzhou First People's Hospital, \\ Nanjing Medical University, Hangzhou, Zhejiang 310006, P.R. China
}

Received September 26, 2016; Accepted March 30, 2017

DOI: $10.3892 /$ etm.2017.4456

\begin{abstract}
The present investigation explored the in vitro culture, isolation and characterization of hair follicle cell differentiation from umbilical cord blood mesenchymal stem cells (MSCs). Flow cytometry was used to obtain MSCs from the isolation and purification of human umbilical cord blood MSCs. Culture suspension of hair follicle organ was centrifuged and the supernatant used in the culture medium of MSCs, and the entire process of induced differentiation was recorded by photomicroscopy. The expression level of surface marker CK15 of hair follicle cells obtained from induced differentiation was detected with immunofluorescence. RT-PCR method was used to further detect the difference in expression of CK15 between hair follicle cells and umbilical cord blood MSCs, and statistical analysis was carried out. CD $44^{+} \mathrm{CD} 29^{+}$double-labeled cells accounted for $50.8 \%$ of all the samples of umbilical cord blood MSCs in this study. The diameter of hair follicle cells differentiated from umbilical cord blood stem cells reached $800 \times 10^{-3} \mathrm{~mm}$ after 3 weeks of cell culture. Based on the detection and colocalization of CK15 expression in induced hair follicle cells, the overlap ratio between CK15 and nuclei reached $83 \%$ in hair follicle cells, which was obviously higher than that in umbilical cord blood stem cells. The difference had statistical significance $(\mathrm{P}<0.05)$. In conclusion, hair follicle cells can be successfully differentiated from umbilical cord blood stem cells by using the supernatant from hair follicle cells. This method can be used for high-speed induced differentiation with high purity, which is promising for clinical application.
\end{abstract}

Correspondence to: Dr Li-Min Wu, Department of Dermatology, Hangzhou First People's Hospital, Nanjing Medical University, 261 Huansha Road, Hangzhou, Zhejiang 310006, P.R. China E-mail: limingwu1973@163.com

Key words: hair follicle cells, mesenchymal stem cells, CK15, differentiation, umbilical cord blood

\section{Introduction}

Stem cells are cells that possess self-renewal capacity and multi-directional differentiation potential in human body. Stem cells are classified by the stage of development into embryonic stem cells (ES) and adult stem cells (ASCs). The differentiation and proliferation of ES is the basis for the development of an organism, namely the development of fertilized eggs into individuals with various tissues and organs. Given that isolation of ES causes damage to the early embryo, this method is not advocated (1). ASC are classified into hematopoietic stem cells and mesenchymal stem cells (MSC) (2). The study of hematopoietic stem cell started in the 1950s. Hematopoietic stem cells mainly originate from bone marrow, adult peripheral blood and umbilical cord blood. Since hematopoietic stem cells can differentiate into mature blood cells (erythrocyte, leukocyte and platelet), they have been widely clinically used in the treatment of hematological diseases (3). In recent years it was found that MSCs also exist in the peripheral blood and umbilical cord blood (4). MSCs can self-proliferate and differentiate into a variety of mature cells, including osteoblast, chondrocyte, adipose cell, myocyte, cardiomyocyte, hepatocyte, neuron, melanocyte, desmocyte and epidermal cells (5-8).

Since the 21st century, MSCs have been used to treat some special diseases related to cell destruction such as neurodegeneration, spinal cord injury, osteoporosis, cardiovascular disease, diabetes, cirrhosis and skin reconstruction. The stem-cell therapy discussed in this study is also focusing on this aspect. Autologous bone marrow was initially the major source of MSCs, and the source has expanded to allogeneic umbilical cord blood in recent years (9-11).

Based on existing studies, this investigation aimed at exploring the molecular control of stem cell differentiation and regulation, and searching for a breakthrough in the directional induced differentiation of stem cells into cellular components of skin tissue such as desmocyte, melanocyte, hair follicle, sebaceous glands and epidermis. Moreover, it is expected that this investigation can provide important theoretical evidence for the treatment of various skin diseases or cosmetic dermatology such as the treatment of melanocyte transplantation for vitiligo, the restoration of damaged skin or scar, the removal of facial wrinkles, breast implants and breast reconstruction. 


\section{Materials and methods}

Materials. Cells: Human umbilical cord blood MSCs: Cyagen Biosciences Inc., Guangzhou, China (art. no. HUXUB-01001). Reagents: Dulbecco's modified Eagle's medium (DMEM) (HyClone, Logan, UT, USA); culture medium for human umbilical cord blood stem cells: Cyagen Biosciences Inc. (art. no. HUXUB-90011); fetal bovine serum (Gibco, Grand Island, NY, USA); $10 \mathrm{mg} / 1$ of insulin, $10 \mathrm{mg} / 1$ of hydrocortisone, $10 \mathrm{mg} / \mathrm{l}$ of glutamine (Sigma, St. Louis, MO, USA); $0.25 \%$ of trypsin (Jrdun Biotechnology Corp., New York, NY, USA); antibodies for flow cytometry: FITC anti-mouse/human CD44 antibody (art. no. 103021; BioLegend, Inc., San Diego, CA, USA); PE anti-human CD29 antibody (art. no. 303003; BioLegend, Inc.).

Preparation of solution. Phosphate-buffered saline (PBS) solution: $8 \mathrm{~g}$ of NaCl, $0.2 \mathrm{~g}$ of $\mathrm{KCl}, 1.44 \mathrm{~g}$ of $\mathrm{Na}_{2} \mathrm{HPO}_{4}$ and $0.24 \mathrm{~g}$ of $\mathrm{KH}_{2} \mathrm{PO}_{4}$ were dissolved in $600 \mathrm{ml}$ of $\mathrm{ddH}_{2} \mathrm{O} ; \mathrm{HCl}$ was used to adjust the $\mathrm{pH}$ to 7.4 and the final volume was brought to 1 liter with $\mathrm{ddH}_{2} \mathrm{O}$; the solution was filtered, autoclaved and stored at room temperature.

Instruments and consumable supplies. $\mathrm{CO}_{2}$ incubator (Thermo Fisher Scientific, Waltham, MA, USA); biosafety cabinet (Suzhou Jinjing Purifying Equipment Technology Co., Ltd., Suzhou, China); pipette (Eppendorf AG, Hamburg, Germany); microscope (Shanghai Caikang Optical Instrument Co., Ltd., Shanghai, China); consumable supplies for cell culture (Corning Inc., Acton, MA, USA).

Isolation and culture of rat hair follicles. The skin in the whisker region of male Wistar rat was acquired under sterile conditions and cleaned several times in PBS containing $100 \mathrm{U} / \mathrm{l}$ of penicillin and $100 \mathrm{mg} / \mathrm{l}$ of streptomycin. Ophthalmic scissors were used to cut through dermis subcutaneously. Microsurgical forceps were used to make blunt dissection of hair follicle and peripheral tissues. The hair follicle was carefully drawn from subcutaneous tissue without damaging the tissues such as hair papilla, and dermal sheath. The adipose tissue around the hair follicle was removed. The complete hair follicles were selected using a microscope. The selected hair follicles were placed in 24-well culture plate, and each well contained one hair follicle. With the addition of $0.5 \mathrm{ml}$ of DMEM culture medium (10\% of fetal bovine serum, $10 \mathrm{mg} / \mathrm{l}$ of insulin, $10 \mu \mathrm{g} / \mathrm{l}$ of hydrocortisone, $2 \mathrm{mmol} / \mathrm{l}$ of glutamine, $100 \mathrm{U} / 1$ of penicillin and $100 \mathrm{mg} / 1$ of streptomycin), the culture was carried out in $5 \% \mathrm{CO}_{2}$ incubator at $37^{\circ} \mathrm{C}$.

Preparation of induction medium. After the first day of hair follicle organ culture, supernatant was collected every day by means of 10-min centrifugation at $150 \mathrm{x}$ g. The supernatant was then sterilized through a $0.22 \mu \mathrm{m}$ filter and stored at $4^{\circ} \mathrm{C}$.

Induced differentiation of human umbilical cord blood MSCs. Human umbilical cord blood MSCs were placed in 6-well culture plate. Filtered induction medium was added after the stem cells completely fused for two days. The culture was carried out in $5 \% \mathrm{CO}_{2}$ incubator at $37^{\circ} \mathrm{C}$. Induction medium was changed every 3 days, and the induction lasted for 21 days.
In control group, culture medium for human umbilical cord blood MSCs was used for cell culture.

Immunophenotypic analysis of umbilical cord blood stem cells. Cell suspension of umbilical cord blood MSCs containing $1 \times 10^{9}$ cells/1 was prepared and fluorescent labeled mouse anti-human antibodies FITC-CD29 and FITC-CD44 were added to the cells. One test tube of cell suspension without added antibodies was used as blank control. Flow cytometry was used to detect the expression of cell surface antigen. The results were analyzed by Image-Pro Plus software (version X; Media Cybernetics, Silver Springs, MD, USA) to calculate the percentage of cells for each surface marker.

$R T$-PCR method. Cells were placed in a $1 \mathrm{ml} \mathrm{TRIzol}{ }^{\circledR}$ to isolate RNA which was stored at $-80^{\circ} \mathrm{C}$ after purification. The PCR amplification of $\mathrm{K} 15$ was done with forward primer sequence: 5'-TTAGCCCTCCACCATTAC-3'; and reverse primer sequence: 5'-TAACTCCACCTCGTTCAG-3'. To amplify GAPDH cDNA, the forward primer sequence: 5'-CACCCA СТССТССАССТTTG-3'; and the reverse primer sequence: 5'-CCACCACCCTGTTGCTGTAG-3' were used.

\section{Results}

Detection and characterization of umbilical cord blood MSCs using flow cytometry. Flow cytometry was used to isolate and purify the samples. Results indicated that CD $44^{+} \mathrm{CD} 29^{+}$ double-labeled cells accounted for $50.8 \%$ of all the samples of umbilical cord blood MSCs as shown in Fig. 1.

Light microscopy images showing the process of induced differentiation of umbilical cord blood MSCs. In order to document the entire process of induced differentiation of stem cells, the entire process was photographed. The results can be seen in Fig. 2A-F.

Identification of hair follicle cells. In order to validate the success of induced differentiation, hair follicle cells were identified. The nucleus was marked with blue fluorescence and CK15 with green fluorescence. The detection and colocalization of surface antigen CK15 expression were carried out using fluorescence microscopy. Results indicated that CK15 in the hair follicle cells was able to colocalize with the nucleus (Fig. 3A-F), and the expression level of CK15 in hair follicle cells was remarkably higher than that in human umbilical cord blood stem cells, and the difference had statistical significance $(\mathrm{P}<0.05$; Fig. $3 \mathrm{G})$. RT-PCR method was used to detect the expression level of CK15 in human umbilical cord blood stem cells and hair follicle cells. It was found that the expression level of CK15 in hair follicle cells was remarkably higher than that in human umbilical cord blood stem cells, and the difference had statistical significance $(\mathrm{P}<0.05$; Fig. $3 \mathrm{H})$. These findings suggested that hair follicle cells were successfully acquired from differentiation.

\section{Discussion}

At present, there are two challenges in the application of stem cells in the clinical treatment: i) The content of MSCs 
A 805

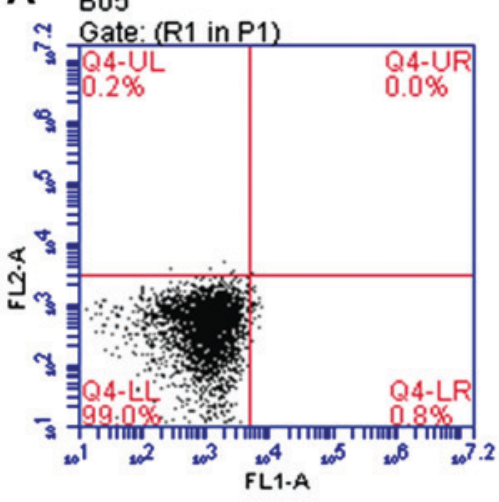

C

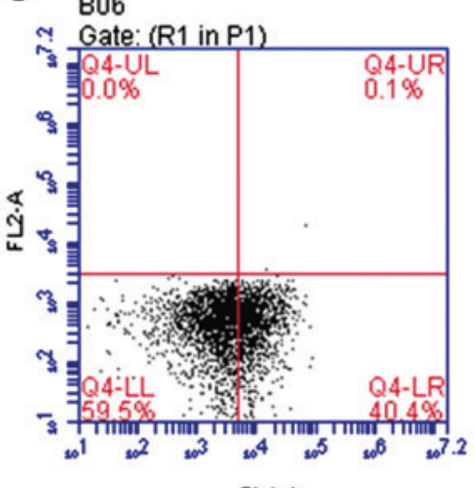

B 807

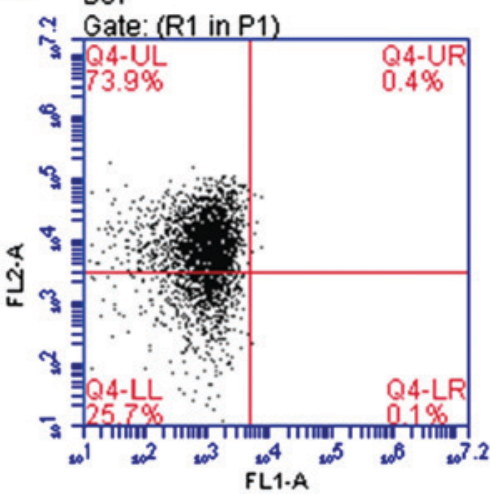

D 808

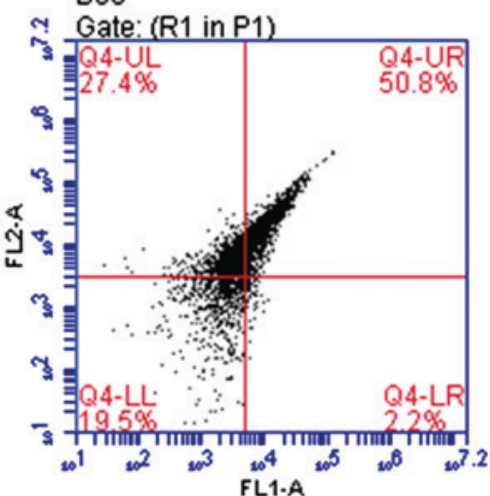

Figure 1. Flow cytometry results of umbilical cord blood mesenchymal stem cells (MSCs). (A) Blank control; (B) CD29 single labeling; (C) CD44 single labeling; (D) CD29+CD44 double labeling. Among all the samples of umbilical cord blood MSCs, CD44 ${ }^{+} \mathrm{CD} 29^{+}$double-labeled cells accounted for 50.8\%.
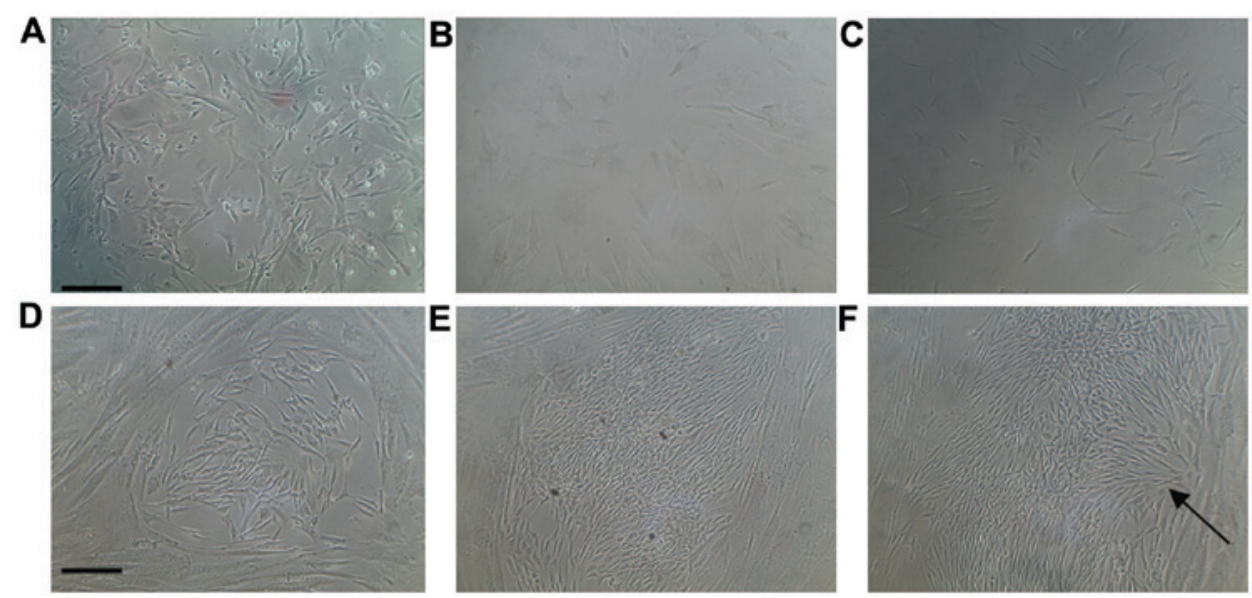

G

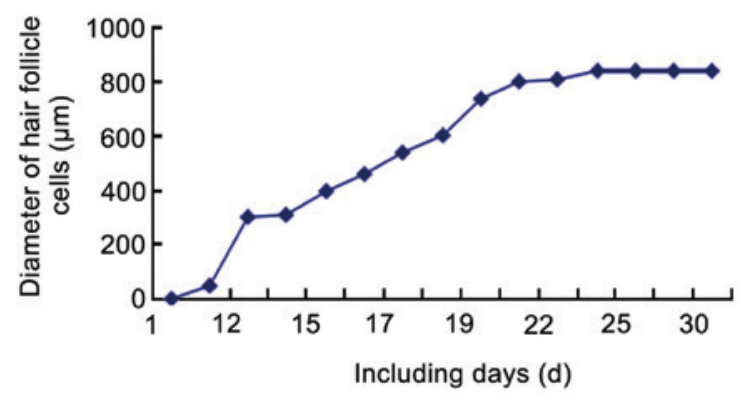

Figure 2. Photomicroscopy using an inverted microscope (magnification, $\mathrm{x} 200$ ) showing the entire process of differentiation of umbilical cord blood stem cells into hair follicle cells (panels A-F). (A) Umbilical cord blood stem cells; (B) fusion of umbilical cord blood; (C) anchorage after digestion; (D) the first week of induction; (E) the second week of induction; (F) the third week of induction. Scale bar, $50 \mu \mathrm{m}$. Arrow points to the proliferation of hair follicle cells; (G) the diameter of hair follicle cells differentiated from umbilical cord blood stem cells reached $800 \mu \mathrm{m}$. 

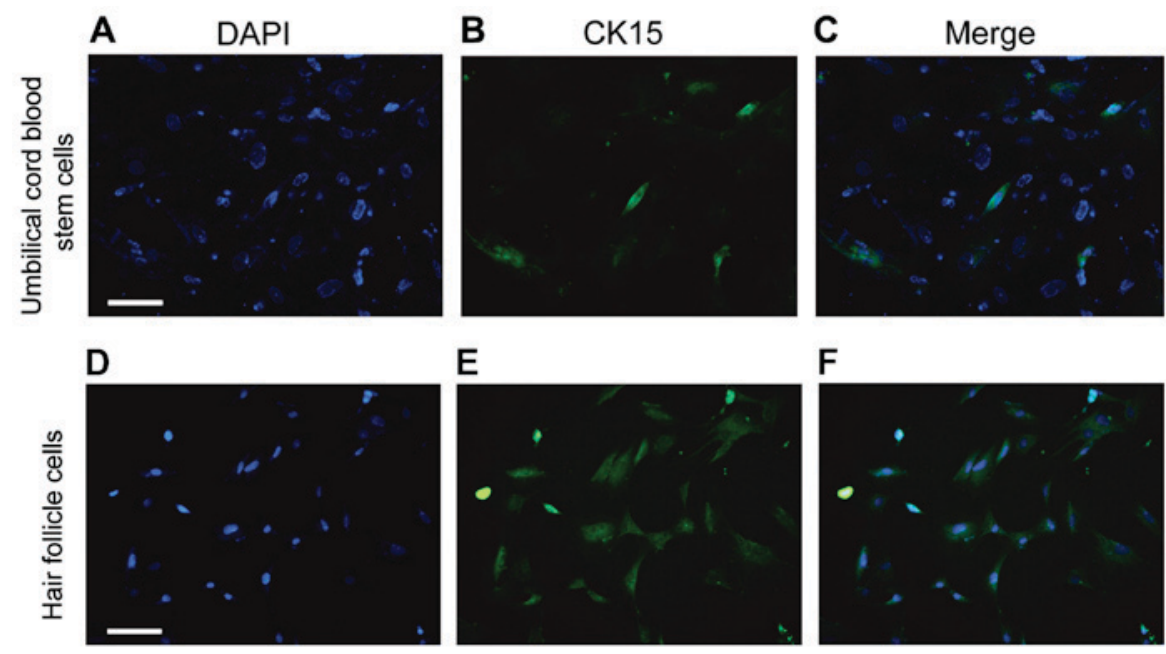

E

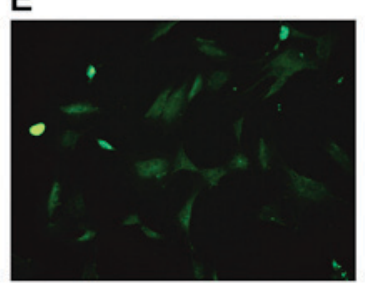

$\mathrm{F}$

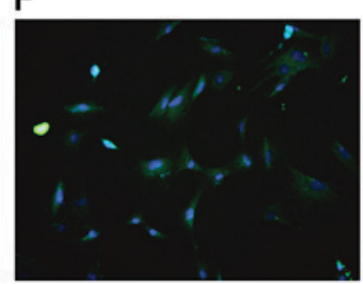

G

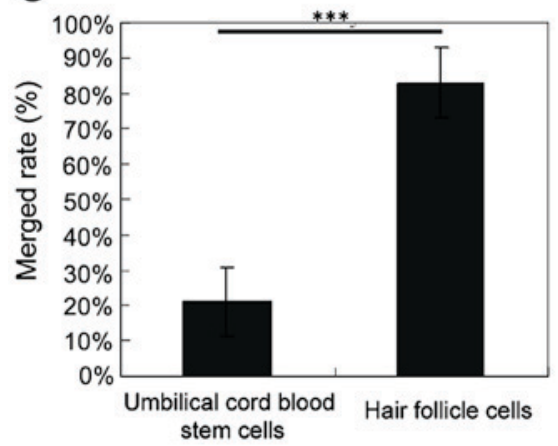

H

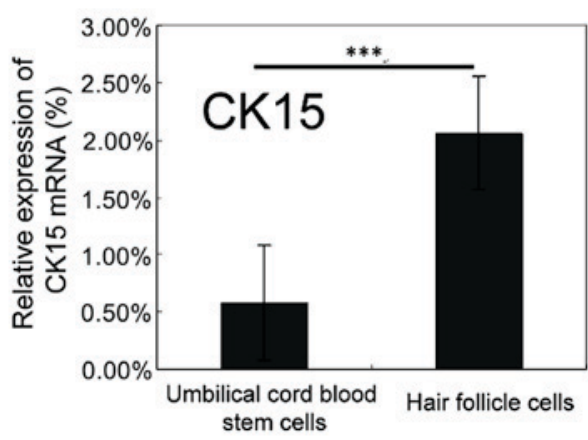

Figure 3. Identification of hair follicle cells using an inverted microscope (magnification, x200). (A-F) The nucleus was marked with blue fluorescence and CK15 with green fluorescence. The detection and colocalization of surface antigen CK15 expression were carried out using fluorescence microscopy. Results indicated that CK15 in the hair follicle cells from differentiation was able to colocalize with nucleus. (G) Using fluorescence microscopy, it was found that the expression level of CK15 in hair follicle cells was remarkably higher than that in human umbilical cord blood stem cells, and the difference had statistical significance $\left({ }^{* * *} \mathrm{P}<0.001\right)$; $(\mathrm{H})$ using RT-PCR method, it was found that the expression level of CK15 in hair follicle cells was remarkably higher than that in human umbilical cord blood stem cells, and the difference had statistical significance $\left({ }^{* * * *} \mathrm{P}<0.001\right)$.

in human body is very low (12). Due to the lack of specific surface marker (antigen) to identify the MSCs, it is difficult to purify this type of cells; ii) in clinical treatment it is essential to culture limited number of MSCs in vitro for effective amplification. Nevertheless, during the amplification of MSCs in vitro, they will spontaneously differentiate and age, which result in the loss of tissue regenerative capacity (13). Moreover, it will also increase the risk of stem cell degeneration and transformation of stem cells into tumor cells. Therefore, these factors strongly inhibit the clinical application of MSCs.

In this study, the techniques for purification and rapid amplification of MSCs were improved, which benefit the further development of induced differentiation and clinical application of stem cells. The advantages of ECM-centered MSC culture system we developed in this study are: i) ECM is completely secreted and built by cells, which is close to the three-dimensional structure of the human body. It can be amplified into numerous MSCs in a short time, and preserve the primitiveness of cells. Therefore, ECM can provide numerous high-performance (all-purpose) MSCs for the study and clinical application of stem cells; ii) since the tissue microenvironment (mainly composed of ECM) determines the direction of MSC differentiation, ECM sourced from different tissues such as muscle, fat, skin and pancreas will effectively induce myocyte, adipose cell, skin cell and islet cell, and provide MSCs with the suitable microenvironment for growth. Because our ECM product source is from the cells in different tissues, the ECM that is composed of cells possesses tissue specificity. These two advantages solved the fundamental problems of quantity and quality of the cells in the clinical application of stem cells in China. Due to the lack of specific marker of hair follicle stem cells at present, the studies of surface marker of hair follicle stem cells mainly focus on the surface markers such as integrin ( $\alpha 6, \beta 1$ and $\beta 4)$, keratin (CK14, CK15 and CK19), CD34, CD200, monoclonal antibody $\mathrm{C} 8 / 144 \mathrm{~B}$, transcription factor p63, CD71 and CX43 (14-16). In this study, we selected CK15 as the surface marker of hair follicle cells to distinguish from umbilical cord blood stem cells. Results indicated that we successfully induced the differentiation of umbilical cord blood into hair follicle cells. Using RT-PCR method to detect the transcription level of CK15, it was also found that the expression level of CK15 in hair follicle cells was remarkably higher than that in human umbilical cord blood stem cells, and the difference had statistical significance $(\mathrm{P}<0.05)$. This is also evidence that by using inducing liquid of hair follicle cells we can successfully induce the differentiation of umbilical cord blood into hair follicle cells. This method can be used for high-speed induced differentiation with high purity, which is promising for clinical application (17-19). 
The finding of transdifferentiation of stem cells not only rewrote the classic concept that the tissue-specific stem cells could only differentiate directionally, but also provided the opportunity for the application of adult-stem-cell therapy. Artificial skin is the earliest tissue-engineered product. Single layer artificial skin was developed in the late 1970s in USA. In 1998, bi-layered artificial skin Apligraft was developed by Organogenesis (USA) and received FDA approval. It is the first human living cell based tissue-engineered product that received FDA approval. Apligraft acts like autologous skin, which possesses the features such as good healing capacity, blood vessel forming capacity, no immune rejection, and so on. It has been used in the treatment of skin burns and venous ulcer, and the cure rate is $>80 \%$ (20).

Although there are a few precedents for adult stem cell therapy, the study and application of ASCs are still in the initial phase. There are still some difficulties in keeping their clonal growth in vitro while inducing them to differentiate into the functional cells required by the treatment. The major reason is that we are not familiar with the microenvironment where the stem cells are present, namely stem-cell niche (21). At present, the stem cell transplantation carried out in laboratories around the world are mainly direct transplantation of stem cells. There exist potential risks for direct transplantation of stem cells in the long-term, because in injured tissue microenvironment, stem cells may generate the cell types that are harmful for tissue repair. Moreover, we cannot exclude the possibility that ASCs may generate tumors. Hence, it is clinically significant to directionally induce the stem cells into required functional cells before transplantation, which is also the emphasis in the future study of MSCs.

In conclusion, using inducing liquid of hair follicle cells, we can successfully induce the differentiation of umbilical cord blood into hair follicle cells. This method can be used for high-speed induced differentiation with high purity, which is promising for clinical application. This induction approach is fast and efficient, and it is able to build up excellent theoretical basis for the future clinical application.

\section{Acknowledgements}

This study was supported by the Hangzhou Science and Technology Development Project (20110833B03).

\section{References}

1. Codinach M, Blanco M, Ortega I, Lloret M, Reales L, Coca MI, Torrents S, Doral M, Oliver-Vila I, Requena-Montero M, et al: Design and validation of a consistent and reproducible manufacture process for the production of clinical-grade bone marrow-derived multipotent mesenchymal stromal cells. Cytotherapy 18: 1197-1208, 2016.

2. Kumar K, Agarwal P, Das K, Mili B, Madhusoodan AP, Kumar A and Bag S: Isolation and characterization of mesenchymal stem cells from caprine umbilical cord tissue matrix. Tissue Cell 48: 653-658, 2016.

3. Del Valle-Echevarria AR, Sanseverino W, Garcia-Mas J and Havey MJ: Pentatricopeptide repeat 336 as the candidate gene for paternal sorting of mitochondria (Psm) in cucumber. Theor Appl Genet 129: 1951-1959, 2016.
4. de Moor JS, Dowling EC, Ekwueme DU, Guy GP Jr, Rodriguez J, Virgo KS, Han X, Kent EE, Li C, Litzelman K, et al: Employment implications of informal cancer caregiving. J Cancer Surviv 11: 48-57, 2017.

5. Sigurjónsson OE, Guðmundsson KO and Guðmundsson S: Mesenchymal stem cells. A review. Laeknabladid 87: 627-632, 2001 (In Icelandic).

6. Maranda EL, Rodriguez-Menocal L and Badiavas EV: Role of mesenchymal stem cells in dermal repair in burns and diabetic wounds. Curr Stem Cell Res Ther 12: 61-70, 2017.

7. Astori G, Amati E, Bambi F, Bernardi M, Chieregato K, Schäfer R, Sella S and Rodeghiero F: Platelet lysate as a substitute for animal serum for the ex-vivo expansion of mesenchymal stem/stromal cells: Present and future. Stem Cell Res Ther 7: 93-100, 2016.

8. Dyrna F, Herbst E, Hoberman A, Imhoff AB and Schmitt A: Stem cell procedures in arthroscopic surgery. Eur J Med Res 21: 29-36, 2016.

9. Jiang LH, Hao Y, Mousawi F, Peng H and Yang X: Expression of $\mathrm{P} 2$ purinergic receptors in mesenchymal stem cells and their roles in extracellular nucleotide regulation of cell functions. J Cell Physiol 232: 287-297, 2017.

10. Morris AD, Chen J, Lau E and Poh J: Domperidone-associated QT interval prolongation in non-oncologic pediatric patients: A review of the literature. Can J Hosp Pharm 69: 224-230, 2016.

11. Kavosi Z, Sarikhani Khorrami M,Keshavarz K, Jafari A,Hashemi Meshkini A, Safaei HR and Nikfar S: Is taurolidine-citrate an effective and cost-effective hemodialysis catheter lock solution? A systematic review and cost-effectiveness analysis. Med J Islam Repub Iran 30: 347-358, 2016.

12. Shi X, Lv S, He X, Liu X, Sun M, Li M, Chi G and Li Y: Differentiation of hepatocytes from induced pluripotent stem cells derived from human hair follicle mesenchymal stem cells. Cell Tissue Res 366: 89-99, 2016.

13. Maruyama CL, Leigh NJ, Nelson JW, McCall AD, Mellas RE, Lei P, Andreadis ST and Baker OJ: Stem cell-soluble signals enhance multilumen formation in SMG cell clusters. J Dent Res 94: 1610-1617, 2015.

14. Son S, Liang MS, Lei P, Xue X, Furlani EP and Andreadis ST: Magnetofection mediated transient NANOG overexpression enhances proliferation and myogenic differentiation of human hair follicle derived mesenchymal stem cells. Bioconjug Chem 26: 1314-1327, 2015.

15. Maleki M, Ghanbarvand F, Reza Behvarz M, Ejtemaei M and Ghadirkhomi E: Comparison of mesenchymal stem cell markers in multiple human adult stem cells. Int J Stem Cells 7: 118-126, 2014.

16. Dong L, Hao H, Xia L, Liu J, Ti D, Tong C, Hou Q, Han Q, Zhao Y, Liu H, et al: Treatment of MSCs with Wntla-conditioned medium activates DP cells and promotes hair follicle regrowth. Sci Rep 4: 5432-5440, 2014.

17. Wu M, Guo X, Yang L, Wang Y, Tang Y, Yang Y and Liu H: Mesenchymal stem cells with modification of junctional adhesion molecule a induce hair formation. Stem Cells Transl Med 3: 481-488, 2014.

18. Wang Y, Liu J, Tan X, Li G, Gao Y, Liu X, Zhang L and Li Y: Induced pluripotent stem cells from human hair follicle mesenchymal stem cells. Stem Cell Rev 9: 451-460, 2013.

19. Gola M, Czajkowski R, Bajek A, Dura A and Drewa T: Melanocyte stem cells: Biology and current aspects. Med Sci Monit 18: RA155-RA159, 2012.

20. DiDomenico L, Landsman AR, Emch KJ and Landsman A: A prospective comparison of diabetic foot ulcers treated with either a cryopreserved skin allograft or a bioengineered skin substitute. Wounds 23: 184-189, 2011.

21. Liang MS and Andreadis ST: Engineering fibrin-binding TGF- $\beta 1$ for sustained signaling and contractile function of MSC based vascular constructs. Biomaterials 32: 8684-8693, 2011. 